\title{
Sistema informático de apoyo a la toma de decisiones en los servicios de restauración de la red hotelera en Cuba
}

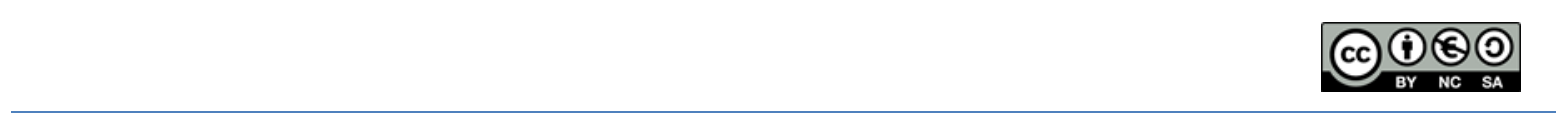

Computer system to support decision-making in the restaurant services of the hotel network in Cuba

Ing. Maday Ynfante Martínez. ${ }^{1}$, Asiel Díaz Vasallo ${ }^{2}$, DrC. Yasser Vázquez Alfonso ${ }^{3} \&$ MSc. Luis Efraín Velasteguí López ${ }^{4}$

Recibido: 10-10-2018 / Revisado: 17-11-2018 /Aceptado: 04-12-2018/ Publicado: 05-01-2019

\begin{abstract}
.
DOI: $\underline{\text { https://doi.org/10.33262/cienciadigital.v3i1.264 }}$

The quality of the services of the hotel network in Cuba is an essential element to guarantee the satisfaction of the tourists that arrive to the country. In this paper, a computer system was developed to detect the level of satisfaction of food and beverage services. In order to do this, the information stored in the databases related to the surveys made to the tourists was analyzed with the purpose of extracting knowledge through the application of data mining techniques.

The system, in addition to managing the data, shows graphical visualizations referring to the behavior of satisfaction indicators, providing analysts with a better understanding of the information. Finally, we discuss issues related to the software proposed in this research and compare the results of the research with those offered by WEKA.
\end{abstract}

Keywords: indicators of satisfaction, data mining.

\section{Resumen.}

La calidad de los servicios de la red hotelera en Cuba es un elemento imprescindible para garantizar la satisfacción de los turistas que llegan al país. En este trabajo se elaboró un sistema informático para detectar el nivel de satisfacción de los servicios

\footnotetext{
${ }^{1}$ Universidad Agraria de la Habana, Facultad Ciencias Técnicas, Mayabeque, Cuba, maday@unah.edu.cu ${ }^{2}$ Universidad Agraria de la Habana, Facultad Ciencias Técnicas, Mayabeque, Cuba, asieldv@unah.edu.cu ${ }^{3}$ Universidad de la Habana, Facultad de Turismo, La Habana, Cuba, yalfos1@gmail.com

${ }^{4}$ Universidad Técnica Cotopaxi, Facultad Sistema, Latacunga, Ecuador, luis.velastegui7838@utc.edu.ec
} 
de alimentos y bebidas. Para ello se analizó la información almacenada en las bases de datos referente a las encuestas realizadas a los turistas, con el propósito de extraer conocimiento mediante la aplicación de técnicas de minería de datos.

El sistema, además de gestionar los datos, muestra visualizaciones gráficas referentes al comportamiento de los indicadores de satisfacción, proporcionándole a los analistas una mayor comprensión de la información. Por último, se abordan temas relacionados con el software propuesto en esta investigación y se comparan los resultados arrojados por el mismo con los ofrecidos por WEKA.

Palabras claves: indicadores de satisfacción, minería de datos.

\section{Introducción}

El turismo es una industria que se ha desarrollado a una velocidad impresionante en las últimas décadas. Motivo por el cual ha llegado a convertirse en una de las primeras fuentes económicas a nivel mundial. Por consiguiente, es de suma importancia a la hora de ofertar servicios turísticos, garantizar un elemento imprescindible: "la calidad". Según Prieto, (2011), su correcta gestión constituye una de las principales ventajas de las organizaciones y las encamina hacia el logro de la mejora continua, como vía para satisfacer las necesidades de sus clientes.

La calidad del servicio y la satisfacción de los usuarios es una de las principales áreas de estudio del comportamiento de los mismos. Esto se debe a que el rendimiento de las organizaciones de servicios es valorado por las propias personas que adquieren y/o utilizan estos bienes de consumo y/o servicios (Quintanilla, 2002). Dentro del sector turístico, los establecimientos de hospedaje y restauración, no sólo constituyen un componente esencial de la oferta, sino que también están experimentando una fuerte competencia.

Romaní (2016) opina que la restauración constituye un eslabón de gran importancia para la industria turística. Es reconocida como uno de los elementos que mayores ingresos reporta al sector hotelero. En los últimos años, los datos de la encuesta nacional de satisfacción del destino revelan que estos servicios no alcanzan los niveles deseados. A su vez, colocan al destino turístico en una situación contraproducente con respecto a los objetivos de calidad establecidos por la institución. Por tal motivo se orienta la necesidad de realizar estudios relacionados con la calidad de los servicios de alimentos y bebidas.

En Espinosa (2010) se evidencian numerosos métodos para la evaluación de la calidad del servicio, que se emplean generalmente como modelos de diagnóstico. Sin embargo, Romaní (2016) afirma que los modelos existentes no contemplan de manera específica una metodología integradora y coherente. Por tal motivo, existe la carencia real de una herramienta que evalúe de forma integral la satisfacción de los turistas respecto a los servicios de alimentos y bebidas en las instalaciones de la red hotelera en Cuba. 
El objetivo de este trabajo es desarrollar un sistema informático que contribuya a la toma de decisiones en los servicios de alimentos y bebidas en las instalaciones de la red hotelera en Cuba.

\section{Metodología}

Para realizar el proceso de minado de datos se empleó la librería $W E K A$ (B. R. R. et al., 2013), la cual presenta un conjunto de técnicas con sus algoritmos asociados. En este proyecto se seleccionaron tres algoritmos. Teniendo en cuenta que el propósito general fue procesar la información desde diversos puntos de vista, la elección de los mismos responde a la aplicación de tres técnicas determinadas (Clustering, Clasificación y Asociación).

En aras de aplicar el Clustering se empleó el método particional $K$-means, con el objetivo de intentar minimizar la varianza total intra-grupo o la función de error cuadrático. En este caso, su uso tributó a la creación de una herramienta de verificación y testeo de la calidad de los servicios de alimentos y bebidas; en particular, del vector de características seleccionado.

Por otro lado, para emplear la Clasificación se recurrió al algoritmo J48, cuya función es crear una descripción eficiente de un conjunto de datos mediante la utilización de un árbol de decisión. Dada una fuente de información consistente, es decir, sin contradicción entre los datos, el árbol resultante describirá el conjunto de entrada a la perfección. Además, el árbol puede ser utilizado para predecir nuevos valores, asumiendo siempre que el conjunto de entrenamiento sobre el cual se trabaja es representativo respecto a la fuente de información original.

La Asociación se puso en práctica con el Apriori, cuya finalidad es descubrir reglas sustentadas probabilísticamente con un intervalo de confianza. Su aplicación posibilitó descubrir patrones significativos dentro de un conjunto de datos, que proporcionan una mejor comprensión del comportamiento general de los indicadores analizados.

Por otra parte, se conformó la base de datos con información suministrada por las encuestas de satisfacción realizadas a los turistas. La misma está compuesta por una tabla principal y cuatro tablas de referencia. La tabla principal contiene la información relevante de la opinión del cliente en sí (Id del cliente, las preferencias por la comida y la bebida, el trato del personal del hotel hacia los mismos entre otros indicadores).

Las tablas de referencia describen la recopilación de determinados campos de la tabla de Opinión del Cliente. Estas son:

- Territorio: contiene la descripción de los territorios que se encuentran en la tabla de Opinión.

- Entidad: que describe las entidades que se encuentran en la tabla de Opinión. 
- Hotel: almacena la información de los hoteles que son utilizados en la tabla Opinión.

- Nacionalidad: contiene la descripción de las nacionalidades que se encuentran en la tabla Opinión.

Es de destacar que se desarrolló una versión de escritorio por petición del usuario final. Para su elaboración se utilizó como entorno de desarrollo integrado NetBeans 8.0, haciendo uso del lenguaje de programación Java. Dicho lenguaje es: orientado a objetos, neutral, portable, robusto, estable, independiente de la plataforma, sencillo y de alto nivel. Cabe señalar que la librería utilizada para realizar el minado de datos fue implementada en este lenguaje, cuestión que facilita la invocación de los métodos contenidos en la misma.

Por otro lado, en aras de mejorar la apariencia de la interfaz gráfica del usuario se empleó JavaFX 8. La misma está compuesta por clases e interfaces escritas en código Java. Para la construcción de la interfaz de usuario estas aplicaciones emplean un lenguaje de marcado declarativo basado en XML, denominado FXML.

Como gestor de bases de datos se utilizó PostgreSQL, el cual está considerado como un gestor muy potente. Cuenta con relaciones objeto-relacional, herencia, los tipos de datos más utilizados, funciones, restricciones, triggers, reglas e integridad transaccional, aunque no llega a ser un gestor completamente orientado a objeto. Permite realizar subconsultas SQL, definir llaves primarias y campos de una tabla "únicos". En el caso de las llaves extranjeras, permite las operaciones de borrar o modificar en cascada a partir de ellas.

Atendiendo a las necesidades del usuario Yfante (2017), estableció como requisitos funcionales:

1. Gestionar los indicadores de restauración.

1.1 Insertar los indicadores de la restauración.

1.2 Modificar los indicadores de la restauración.

1.3 Eliminar los indicadores de la restauración.

2. Gestionar usuarios.

2.1 Insertar usuarios.

2.2 Modificar usuarios.

2.3 Eliminar usuarios.

3. Autenticarse.

4. Generar reportes sobre los indicadores de la restauración.

4.1 Reporte en relación a los productos.

4.1.1 Reporte de aceptación de los índices de calidad de los productos por hotel.

4.1.2 Reporte de reglas de asociaciones entre los índices de calidad de los productos.

4.2 Reporte en relación a la bebida.

4.2.1 Reporte de aceptación de los índices de calidad de la bebida por hotel. 
4.2.2 Reporte sobre la preferencia de la bebida.

4.3 Reporte en relación al personal.

4.3.1 Reporte de aceptación de los índices de calidad referentes al personal de servicio por hotel.

4.3.2 Reporte de reglas de asociaciones entre los índices de calidad concernientes al personal de servicio.

4.4 Reporte en relación a la instalación.

4.4.1 Reporte de aceptación de los índices de calidad referidos a la instalación.

4.4.2 Reporte de reglas de asociaciones entre los índices de calidad pertenecientes a la instalación.

4.5 Reporte en relación a la comida.

4.5.1 Reporte sobre la preferencia de la comida.

4.6 Índices de satisfacción general de bebida.

4.7 Índices de satisfacción general de alimentos.

4.8 Reporte sobre las reglas de asociación entre comida y bebida.

5. Graficar el comportamiento de los indicadores de la restauración mediante la agrupación en clústeres de:

5.1 Índices en relación a la bebida.

5.2 Índices en relación al personal.

5.3 Índices en relación a la instalación.

5.4 Índices en relación a la comida.

5.5 Índices de satisfacción general de bebida.

5.6 Índices de satisfacción general de alimentos.

Como Requisitos no funcionales se definió:

Interfaz externa o apariencia:

- La interfaz debe ser sencilla y amigable para facilitar el trabajo con el software, pues el sistema brindará servicios tanto a usuarios familiarizados con ambientes informáticos como a otros no familiarizados.

Rendimiento:

- El sistema debe ser lo más estable y fiable posible alcanzando alta velocidad de respuesta a las peticiones de los usuarios.

Soporte:

- El sistema será instalado y configurado por los especialistas del Departamento de Informática de la Universidad Agraria de La Habana, quienes se encargarán de darle mantenimiento durante su período de prueba.

Extensibilidad: 
- El sistema debe ser capaz de permitir que se le agreguen nuevos módulos o cambios, para ello la programación de sus componentes se realizará lo más independiente posible.

Mantenimiento:

- Debe dar facilidad de mantenimiento, y desarrollarse los más sencilla y eficientemente posible, para que en un futuro pueda ser atendido por otros grupos de trabajo.

Portabilidad:

- El sistema debe ser utilizado bajo los sistemas operativos Windows o Linux, por lo que su desarrollo debe realizarse haciendo uso de lenguaje y tecnologías capaces de brindar este soporte.

Software:

- PostgreSQL como sistema gestor de base de datos relacionales.

- Java Development Kit 8 Update 20.

Hardware:

- Como mínimo se requiere de un ordenador Pentium IV con 1 GB de RAM y un microprocesador a $2.0 \mathrm{Ghz}, 120 \mathrm{~Gb}$ de disco duro.

\section{Resultados}

Los resultados expresan el comportamiento que toman los indicadores recogidos en las encuestas al aplicarle las técnicas de minería de datos, los cuales se ven reflejados en las gráficas para un mejor uso de la información.

Con el propósito de estudiar el nivel de inestabilidad que presentan los indicadores de las instancias (registros) analizadas, se empleó el gráfico de puntos o scatter (ver gráfica 1). Su uso contribuyó a la visualización del comportamiento de las opiniones recopiladas; estableciendo agrupaciones mediante criterios de similitud existentes.

Gráfica 1: Agrupación en clúster.

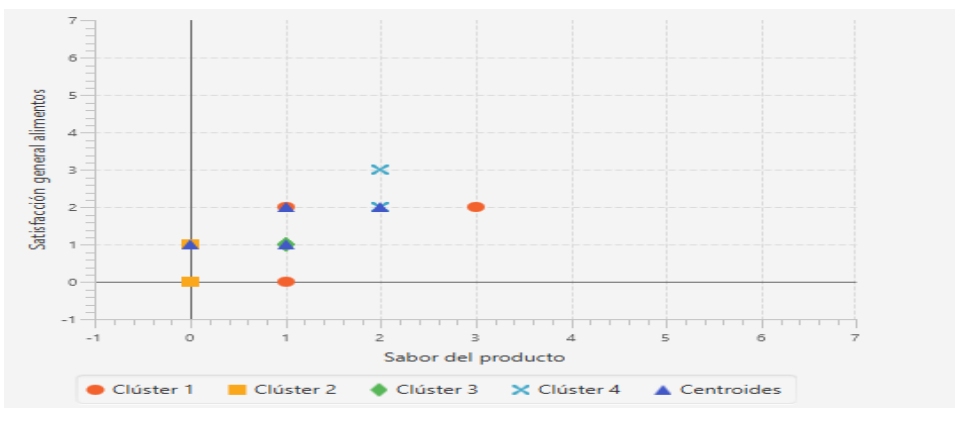

Fuente: Elaboración propia. 
La misma nos revela que se establecieron cuatro agrupaciones. En cuanto al clúster número uno (de color naranja), se observa una variabilidad tanto en la satisfacción general de los alimentos, como en el sabor del producto. Por otra parte, el clúster dos (de color amarillo mostaza) señala que no existe inestabilidad con respecto al sabor del producto mientras que para la satisfacción general de los alimentos la hay. En el clúster tres (de color verde) todos los indicadores son análogos. Por otro lado, en el clúster cuatro (de color azul) existe variabilidad con respecto a la satisfacción general de los alimentos.

Para visualizar la distribución de un determinado elemento según las variables de los atributos que se relacionan se utilizan los gráficos de barras o Bar Chart (ver gráfica 2) los cuales permiten mostrar la cantidad de instancias agrupadas por clúster.

Gráfica 2: Gráfico de barras

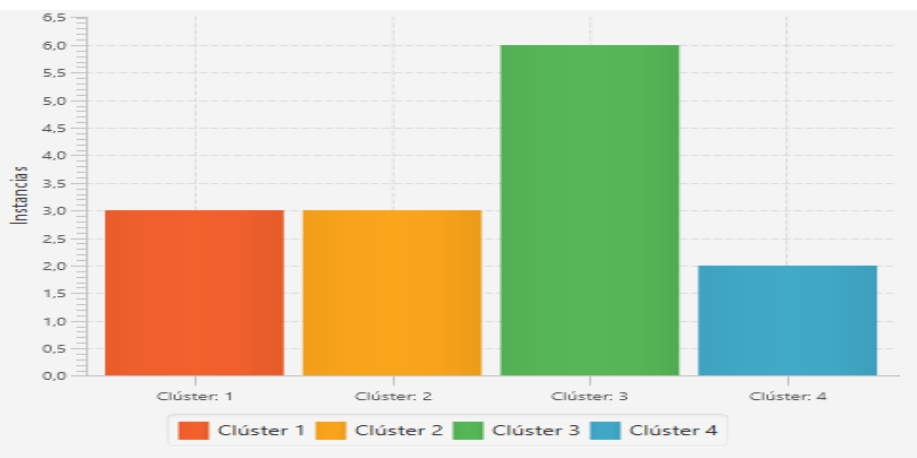

Fuentes: Elaboración propia.

En dicha gráfica se puede apreciar que el clúster tres presenta el mayor número de indicadores (6). A su vez, el clúster cuatro, refleja la menor proporción registrada. Mientras que el clúster 1 y 2 presentan la misma cantidad de indicadores (3).

Para mostrar las combinaciones de valores de los atributos (indicadores) que acontecen más frecuentemente en un gran volumen de datos, se utilizan las Reglas de Asociación. Las mismas permiten conocer el comportamiento general de la información para utilizarlo a la hora de tomar decisiones (ver figura 1).

Figura 1: Reglas de Asociación. 


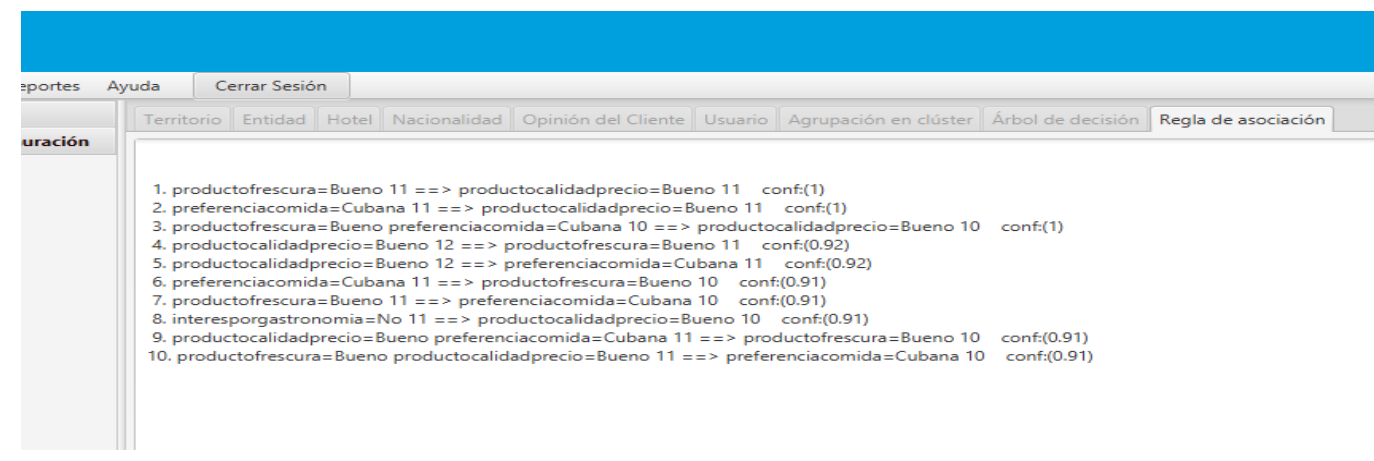

Fuente: Elaboración propia.

En la primera regla se observa que 11 personas expresan que la frescura del producto es Bueno lo cual implica que el precio de calidad del producto es Bueno para 11 personas también, con un nivel de confiabilidad del 100\% (conf:(1)).

Un árbol de decisión es un conjunto de reglas de asociación organizadas de tal manera que la decisión final o acción a tomar se puede determinar siguiendo las mismas desde la raíz hasta alguna de sus hojas. Con la finalidad de catalogar como se están efectuando los servicios que se prestan a los turistas, se estudian un grupo de condiciones para predecir el camino a tomar para mejorar o hacer de excelencia los mismos.

Para efectuar la predicción se recurrió al algoritmo J48 utilizando como variable en estudio el indicador que se desea analizar (ver figura 2).

Figura 2: Árbol de decisiones.

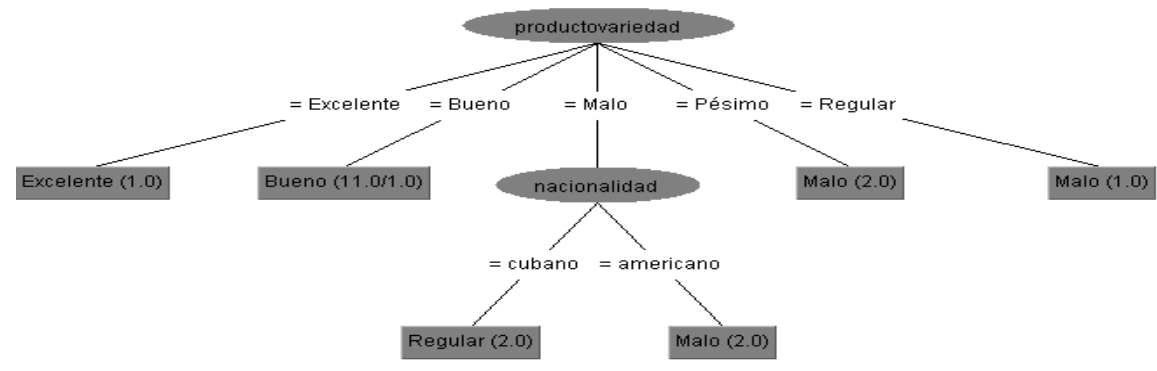

Fuente: Elaboración propia.

Este caso responde a la variable objetivo satisfacción general de los alimentos (Indicador de la encuesta). Las ramas que surgen de cada nodo representan los estados posibles que puede tomar el atributo del nodo; y hojas, en donde se muestra la clasificación a cada clase (en este caso Excelente, Bueno, Regular y Malo). En las hojas se muestra la cantidad total de registros clasificados y separados con una barra, la cantidad de registros mal clasificados (si los hubiera). 
A continuación, se enunciarán las reglas resultantes del árbol representado anteriormente:

Regla 1:

Si la variedad del producto es Excelente

Entonces la satisfacción general de los alimentos es Excelente (1)

Regla 2:

Si la variedad del producto es Bueno

Entonces la satisfacción general de los alimentos es Bueno (11/1)

Regla 3:

Si la variedad del producto es Malo y la nacionalidad es cubano

Entonces la satisfacción general de los alimentos es Regular (2)

Regla 4:

Si la variedad del producto es Malo y la nacionalidad es americano

Entonces la satisfacción general de los alimentos es Malo (2)

Regla 5:

Si la variedad del producto es Pésimo

Entonces la satisfacción general de los alimentos es Malo (2)

Regla 6:

Si la variedad del producto es Regular

Entonces la satisfacción general de los alimentos es Malo (1)

Por otro lado, las matrices de confusión permiten entender cuál es el error que comete un árbol de decisión al intentar clasificar todos los registros. Para este caso la matriz de confusión resultante se muestra en la tabla 1 .

Tabla 1: Matriz de confusión.

\begin{tabular}{l|cccc}
\multicolumn{5}{c}{ Clasificado como } \\
\hline & $\mathrm{a}$ & $\mathrm{b}$ & $\mathrm{c}$ & $\mathrm{d}$ \\
$a=$ Excelente & 0 & 2 & 0 & 0 \\
$b=$ Bueno & 0 & 9 & 1 & 0 \\
$c=$ Regular & 0 & 1 & 2 & 2 \\
$d=$ Malo & 0 & 0 & 1 & 1
\end{tabular}

Fuente: Elaboración propia.

Si la clasificación hubiera sido perfecta se podrían encontrar únicamente elementos en la diagonal. De no ser así, como es el caso la clasificación de los hechos se ve afectada. 


\section{Experimentación.}

Para demostrar la veracidad de los resultados, se establecieron comparaciones entre las salidas arrojadas por la aplicación (los resultados de las tres técnicas utilizadas, Clustering, Clasificación y Asociación) y las que proporciona la herramienta WEKA.

La figura 3 ofrece una representación gráfica de la agrupación de las distintas opiniones de los clientes y la salida que efectuó la herramienta WEKA utilizando los mismos parámetros que se manejaron para la aplicación.

Figura 3: Gráficos de clúster.

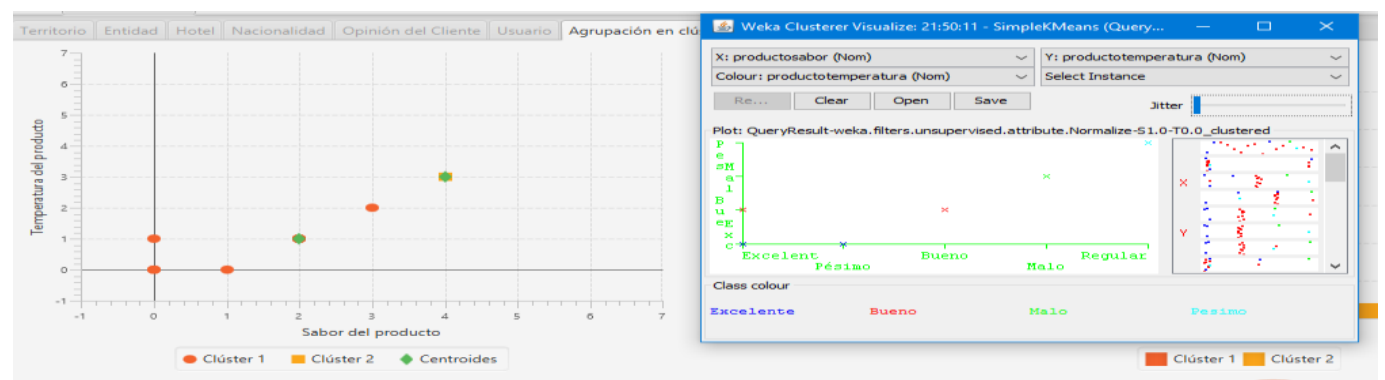

Fuente: Elaboración propia.

La figura muestra la similitud de los resultados que arroja la aplicación con los de la herramienta WEKA. A la izquierda se evidencia la información generada por la variante propuesta en este trabajo. La cuál muestra los clústeres que se construyeron y nos indica que tan variable son los indicadores que conforman un mismo clúster. A la derecha se ofrece la salida de la herramienta para ratificar la información que aporta el software. En este caso se puede apreciar que los resultados obtenidos son equivalentes.

Las asociaciones determinadas por el algoritmo Apriori proporcionan un conjunto de reglas generadas a partir de la frecuencia de ocurrencias, que se establecen en las relaciones de precedencia entre los indicadores. En la figura 4 se muestran las asociaciones obtenidas con WEKA, mientras que en la figura 5 se visualiza las reglas generadas por la aplicación a partir del mismo conjunto de datos. De esta forma se determinan los hechos que anteceden y los que preceden, formando las relaciones con un intervalo de confianza dado.

Figura 4: Reglas obtenidas por la herramienta WEKA 


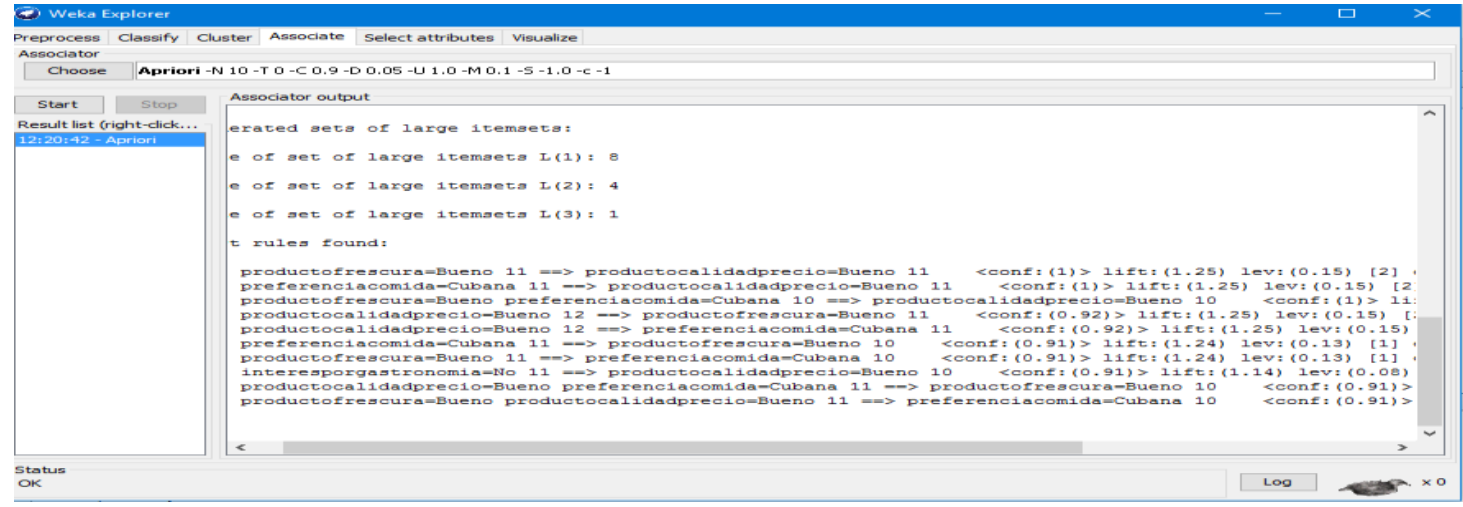

Fuente: Elaboración propia.

Figura 5: Reglas obtenidas por la aplicación.

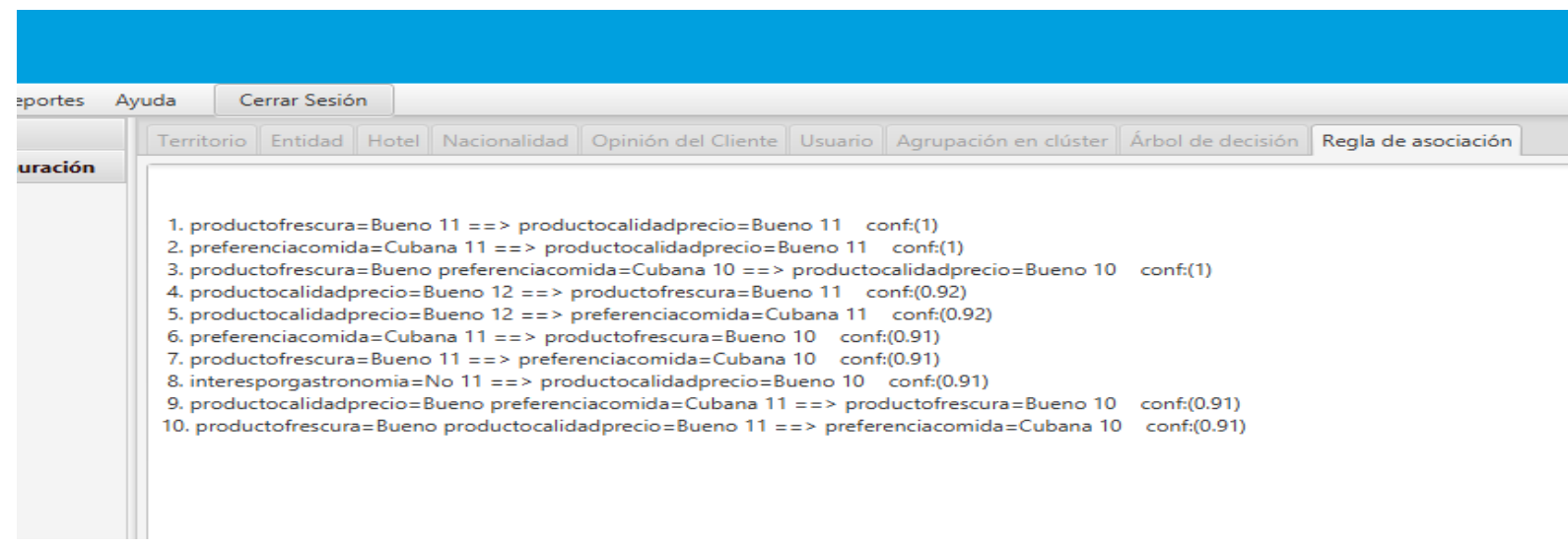

Fuente: Elaboración propia.

Por otro lado, la clasificación establecida por el algoritmo J48 proporciona una representación estructural donde se relacionan distintos indicadores atendiendo a diversos criterios (ver figura 6).

Figura 6: Árbol de decisiones de la aplicación. 


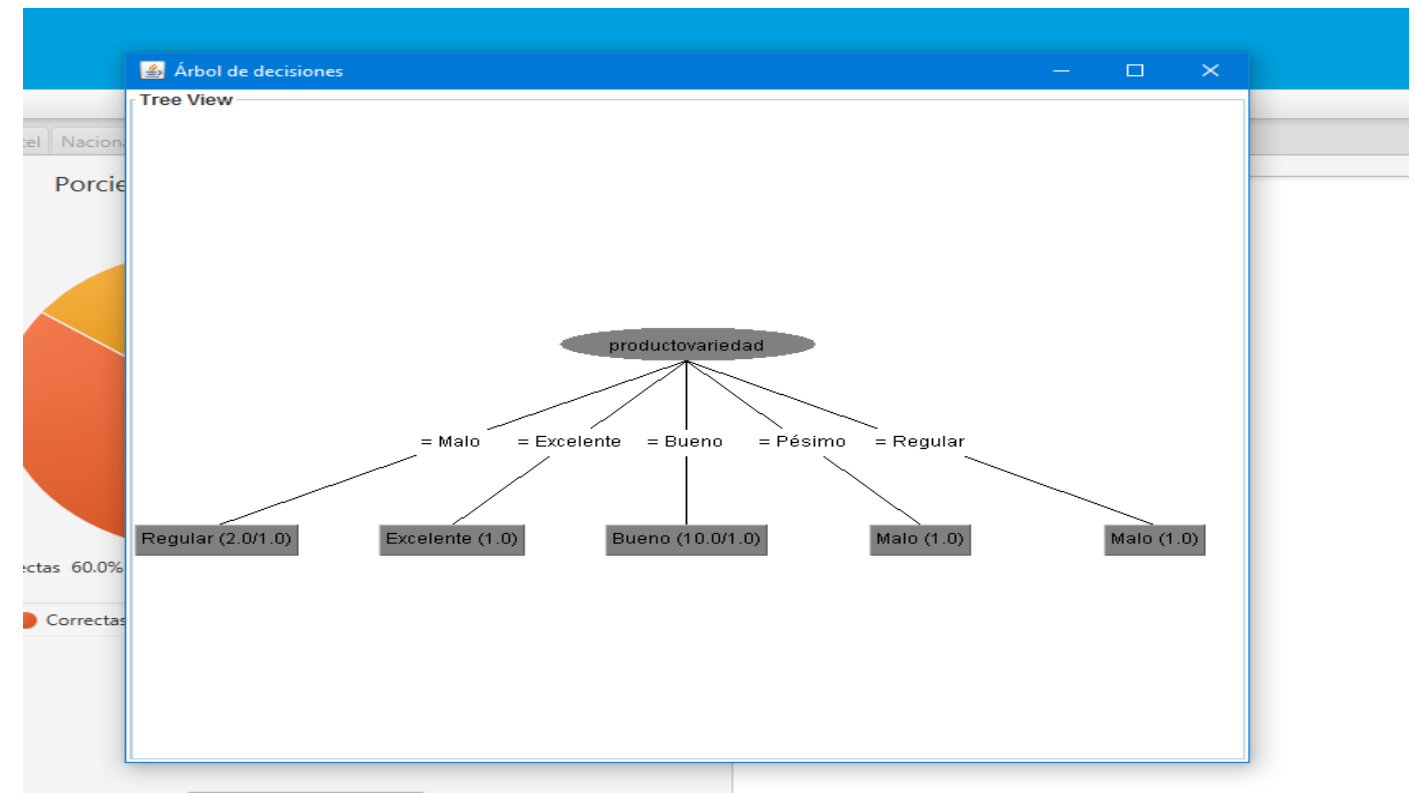

Fuente: Elaboración propia.

La figura anterior corresponde a una gráfica generada por el sistema, representando un árbol de decisión que toma en cuenta una variable destino u objetivo. En este caso se tomó el indicador "satisfacción general de los alimentos" como variable objetivo. A partir de la cual, se selecciona la mejor partición y se comienza a construir la jerarquía de clases hasta llegar al fin.

En la figura 7 se refleja la visualización proporcionada por $W E K A$, haciendo uso de dicho algoritmo. Como se puede observar, el árbol de decisión representado coincide con el generado por la aplicación propuesta en esta investigación.

Figura 7: Árbol de decisiones de WEKA.

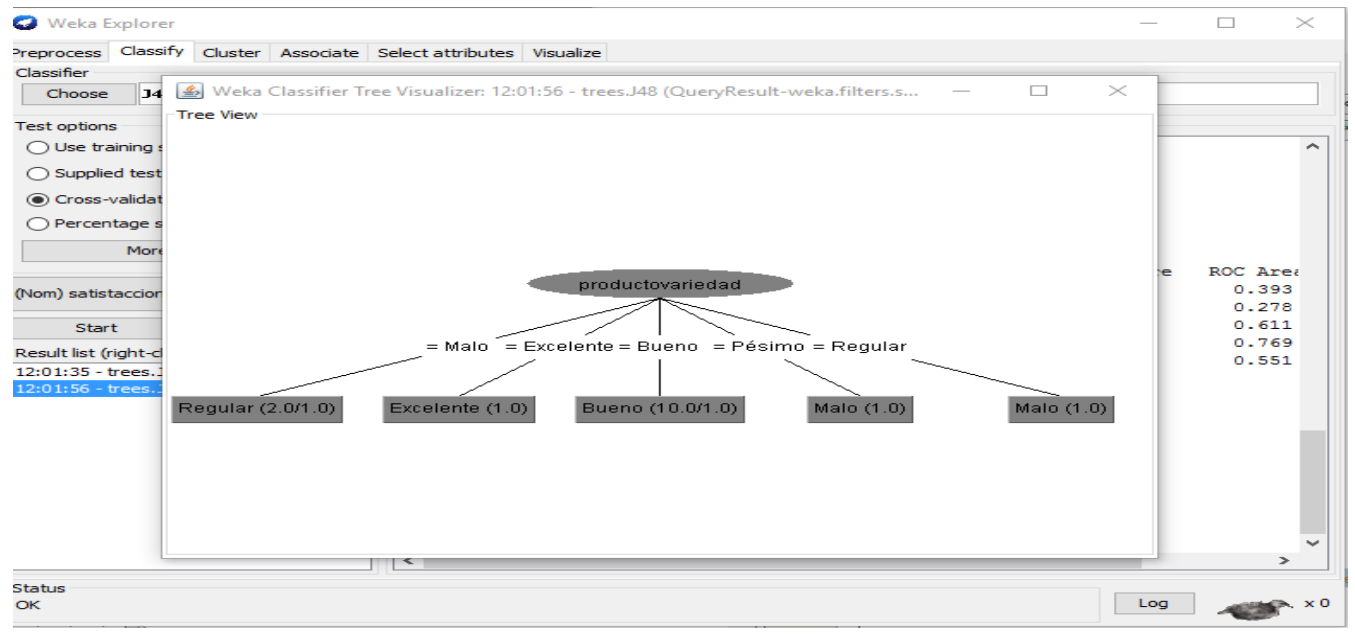


Fuente: Elaboración propia.

El desarrollo de esta investigación cumplió con las expectativas del Grupo Empresarial Palmares y el grupo de desarrolladores, alcanzando los siguientes beneficios tangibles e intangibles.

\section{Beneficios tangibles:}

1. Contribuirá a la toma de decisiones.

2. Aumento de la calidad del trabajo.

3. Disminuye el tiempo de procesamiento.

\section{Beneficios intangibles:}

1. El desarrollo del sistema ha posibilitado al desarrollador conocimientos en las tecnologías utilizadas.

2. Fácil procesamiento de la información.

3. Permite a la entidad contar con una herramienta de análisis de los indicadores de satisfacción de los turistas que consumen el servicio.

Los resultados alcanzados en esta investigación complementan a los resultados obtenidos por: Prieto (2011), Lottis (2015), Espinosa (2015), Romaní, B. y Calás, D (2015), Niebla (2016) y Mesa (2017), ya que se propone una herramienta informática con algoritmos matemáticos que permite el análisis de la satisfacción del cliente de manera inteligente.

\section{Conclusiones}

- Se elaboró una aplicación informática que permite el análisis de la información relevante, siendo esta una herramienta amigable, sencilla, segura y eficiente.

- La integración de los algoritmos matemáticos, con su implementación computacional constituyen una herramienta para el análisis de la información en las instalaciones del MINTUR.

- Los resultados obtenidos tras aplicar los algoritmos de K-means, J48 y Apriori proporcionaron al usuario una mejor interpretación del comportamiento de los indicadores.

- Durante la fase de pruebas realizadas al sistema, se alcanzaron los resultados esperados.

\section{Referencia bibliográfica.}

B. R. R. et al ( 2013). WEKA Manual for Version 3-7-8. New Zealand: University of Waikato.

Espinosa Manfugás, J. M (2010). Gestión de la Restauración.. Ciudad de La Habana: s.n. 
Espinosa, Y (2015). Evaluación de la satisfacción del cliente nacional en la Empresa Cubatur Habana. Trabajo de diploma. Facultad de Turismo. Universidad de la Habana. La Habana, Cuba.

Figueroa, R. G., Solís, C. J. \& Cabrera, A. A (2012). Metodologías tradicionales vs. Metodologías ágiles, Loja: s.n.

GmbH, R.-I (2009). Rapidiner 4.4. User Guide. s.1.:s.n.

Hernández, O. J., Ramírez, Q. J. \& Ferri, R. C (2013). Introducción a la Minería de datos. s.l.:Prentice Hall.

H. M., F. E. \& W. I. H (2011). Practical Data Mining.Tutorial 1:Introduction to the WEKA Explorer. Waikato: University of Waikato.

Yfante, M (2017). Sistema informático de apoyo a la toma de decisiones en los servicios de restauración de la red hotelera en Cuba. Trabajo de Diploma en opción al Título de Ingeniería en Informática. Facultad de Ciencias Técnicas. Universidad Agraria de la Habana. Cuba.

Lottis, K. D (2015). Evaluación de la satisfacción del cliente con los servicios de alimentos y bebidas en instalaciones del Grupo Cubanacán en La Habana. Trabajo de Diploma en opción al título de Licenciado en Turismo. Facultad de Turismo. Universidad de La Habana. La Habana. Cuba.

Meléndez, C (2014). Calidad de servicio al cliente y su incidencia en la rentabilidad de las empresas del rubro Restaurantes - Pollerías. Tarapoto. Perú.

Mesa, L R (2017). "Diseño de un instrumento para la determinación de las expectativas de los turistas sobre el servicio de alimentos y bebidas en el destino Cuba".

Niebla. M (2016). "Evaluación del nivel de satisfacción de los clientes que visitan el Club Temático Jazz Café”. Trabajo de Diploma en opción al Título de Licenciado en Turismo. Facultad de Turismo. Universidad de la Habana. Cuba.

Prieto, H. J (2011). Diagnóstico de la Calidad en el Restaurante Buffet "El Tucán" del Hotel Cubanacán Copacabana. Tesis en opción al título de Licenciado en Turismo.. Ciudad de La Habana: s.n.

Quintanilla, I (2002). Psicología Social del Consumidor.. Valencia: s.n.

Romaní Bendig, M. B ( 2015). Metodología para la evaluación de la calidad de los servicios de alimentos y bebidas de la red hotelera del destino Cuba, La Habana: s.n.

Romaní, B. y Calás, D (2015).“La satisfacción del cliente y su medición. Un eslabón fundamental para la garantía de la calidad de los servicios turísticos”. Revista Mundo Turístico. No agosto-diciembre. RNPS 0492/ ISSN 1727-1568. 


\section{Para citar el artículo indexado.}

Ynfante M., Díaz A., Vázquez Y. \& Velasteguí E L. (2019). Sistema informático de apoyo a la toma de decisiones en los servicios de restauración de la red hotelera en Cuba. Revista electrónica Ciencia Digital 3(1), 92-106. Recuperado desde: http://cienciadigital.org/revistacienciadigital2/index.php/CienciaDigital/article/view/264/61 $\underline{2}$

\section{¿Ciencia}

El artículo que se publica es de exclusiva responsabilidad de los autores y no necesariamente reflejan el pensamiento de la Revista Ciencia Digital.

El articulo queda en propiedad de la revista y, por tanto, su publicación parcial y/o total en otro medio tiene que ser autorizado por el director de la Revista Ciencia Digital.
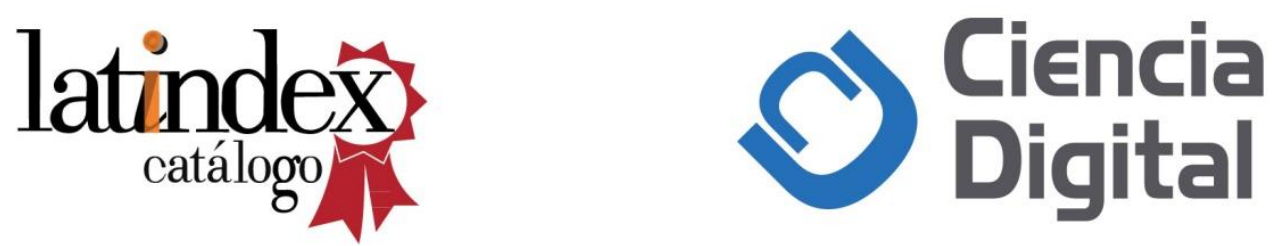